Optimal eye movement strategies : a comparison of neurosurgeons gaze patterns when using a surgical microscope

\title{
Eivazi, Shahram
}

2017-06

Eivazi , S , Hafez , A , Fuhl , W , Afkari , H , Kasneci , E, Lehecka , M \& Bednarik , R 2017 , ' Optimal eye movement strategies : a comparison of neurosurgeons gaze patterns when using a surgical microscope ' , Acta Neurochirurgica , vol. 159 , no. 6 , pp. 959-966 . https://doi.org/10.1007/s00701-

http://hdl.handle.net/10138/235538

https://doi.org/10.1007/s00701-017-3185-1

unspecified

publishedVersion

Downloaded from Helda, University of Helsinki institutional repository.

This is an electronic reprint of the original article.

This reprint may differ from the original in pagination and typographic detail.

Please cite the original version. 


\title{
Optimal eye movement strategies: a comparison of neurosurgeons gaze patterns when using a surgical microscope
}

\author{
Shahram Eivazi ${ }^{1}$ (D) Ahmad Hafez $^{2} \cdot$ Wolfgang Fuhl $^{3} \cdot$ Hoorieh Afkari $^{1}$ • \\ Enkelejda Kasneci $^{3} \cdot$ Martin Lehecka $^{2} \cdot$ Roman Bednarik ${ }^{1}$
}

Received: 10 February 2017 / Accepted: 5 April 2017 /Published online: 19 April 2017

(C) Springer-Verlag Wien 2017

\begin{abstract}
Background Previous studies have consistently demonstrated gaze behaviour differences related to expertise during various surgical procedures. In micro-neurosurgery, however, there is a lack of evidence of empirically demonstrated individual differences associated with visual attention. It is unknown exactly how neurosurgeons see a stereoscopic magnified view in the context of micro-neurosurgery and what this implies for medical training.

Method We report on an investigation of the eye movement patterns in micro-neurosurgery using a state-of-the-art eye tracker. We studied the eye movements of nine neurosurgeons while performing cutting and suturing tasks under a surgical microscope. Eye-movement characteristics, such as fixation (focus level) and saccade (visual search pattern), were analysed.

Results The results show a strong relationship between the level of microsurgical skill and the gaze pattern, whereas more expertise is associated with greater eye control, stability, and focusing in eye behaviour. For example, in the cutting task, well-trained surgeons increased their fixation durations on the operating field twice as much as the novices (expert, $848 \mathrm{~ms}$; novice, $402 \mathrm{~ms}$ ). Conclusions Maintaining steady visual attention on the target (fixation), as well as being able to quickly make eye jumps
\end{abstract}

S. Eivazi and A. Hafez contributed equally to this project

Shahram Eivazi

seivazi@cs.joensuu.fi

1 School of Computing, University of Eastern Finland, 80101 Joensuu, Finland

2 Department of Neurosurgery, Helsinki University Central Hospital, Helsinki, Finland

3 Department of Computer Science, University of Tübingen, Tübingen, Germany from one target to another (saccades) are two important elements for the success of neurosurgery. The captured gaze patterns can be used to improve medical education, as part of an assessment system or in a gaze-training application.

Keywords Medical practice - Visual attention · Microsurgery $\cdot$ Surgical training $\cdot$ Neurosurgery

\section{Introduction}

Using a microscope in neurosurgery involves a broad range of highly complex and technical skills. One of the skills is to keep the eye focused while working in a narrow field with different magnifications for a prolonged time. Microneurosurgery requires a certain level of indirect eye-hand coordination, and any imprecise movement can have life-critical implications for patients.

In response to these complexities, neurosurgeons undergo a long training period and have to continue practicing manipulating instruments and objects under the microscope. Harmonic movement allows neurosurgeons to achieve efficient and suitable automatic reaction during operating procedures [4-6, 30, 31].

We aim to capture and understand visual information processes involved in micro-neurosurgery as a step toward modelling human information processing in this domain. The particular property that we explore deals with understanding how an experienced neurosurgeon effectively moves his/ her eyes to follow the procedure and avoid obstacles while safely moving the instrument through delicate structures.

We hypothesised that there is a difference among neurosurgeons' gaze patterns concerning expertise and experience, and time of using the microscope. However, task-specific 
challenges play an important role in disturbing eye-hand coordination while working under the microscope.

\section{Eye tracking in medical training}

Previous studies in different medical fields have proved that eye gaze patterns depend on experience [13, 19, 33, 36]. Research in interpreting radiology images is where most of the visual attention studies have taken place [24, 25, 29]. These studies support the hypothesis that visual searches in medical images begin with a global scan to filter abnormal areas from normal or noise features.

There is a large and growing body of eye-tracking studies investigating gaze patterns in image-guided surgery, namely laparoscopic surgeries $[19,22,27,40]$. For instance, Law et al. [27] and Wilson et al. [40] showed that novices are more varied in their gaze pattern and often look at the instrument tip, whereas experts tend to maintain their gaze on the target while manipulating the surgical instrument.

Regarding live surgeries, Atkins et al. [3] and Tomizawa et al. [37] studied expertise behavioural differences in the operating room (OR). Tomizawa et al. reported that expert perfusionists tend to disperse their attention more widely than novices. Moreover, the experts direct their fixations to a relatively large number of key information groups during surgery.

In neurosurgery, Eivazi et al. [9] studied the gaze behaviour of expert and novice surgeons in an off-line situation outside the OR. The results showed differences in the way that expert and novice surgeons look on the surgery images and in attention control; experienced surgeons were able to focus for prolonged times at the target points.

In this paper, we present an original contribution extending prior work reported in Acta Neurochirurgica [10] and other scientific communities $[1,2,9,11,23]$. In the following, we describe experiment design, the procedure of recording eye movements and data analysis.

\section{Method}

The experiment was conducted in the Neurosurgery Department of the Helsinki University Central Hospital, using a Leica neurosurgical microscope (SMED Model SY2.2), outside an OR (Fig. 1).

\section{Experiment design}

To provide a repeatable controlled setting, we simulated a vessel wall suturing procedure. We divided our experiment into two tasks. In the first task, participants were asked to cut precisely along the lines drawn on the top of a latex glove sheet. For the second task, we asked them to suture an already cut latex glove. They were required to distribute six stitches with five knots on each side of the model (in total, 12 stitches).

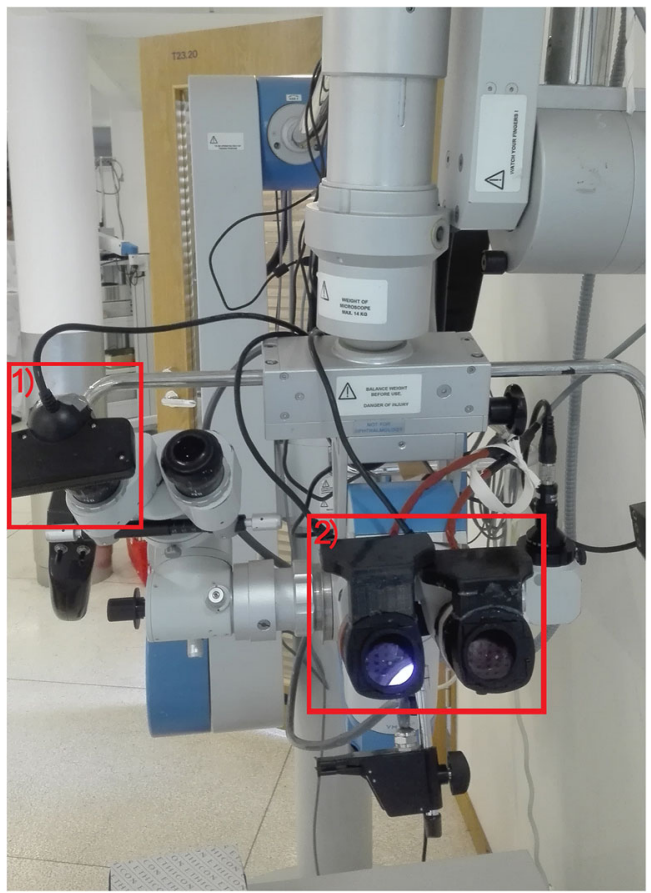

Fig. 1 Experimental setup and microscope outside the OR. 1 Eye tracker and 2 scene camera used in this study

We designed our model (Fig. 2) by reviewing the microsurgery literature $[5,12,26]$. We developed this shape with expert surgeons, using a millimetre paper, taking into account the handinstrument orientation skill aspect of the neurosurgical procedures.

The two outer circles ( $A$ and $B$ in Fig. 2) represent the field of view under the microscope when using two different magnifications lower magnifications for cutting and higher for suturing. We chose a 15 -mm radius for the first circle $(A)$ to be used with fivepoint magnification. The second circle $(B)$ has a $10-\mathrm{mm}$ radius, which covers the microscope view with seven-point magnification.

To keep the model fixed (avoid sagging, hogging and movement), we designed a 2-mm radius circle in the centre of the model ( $D$ in Fig. 2). Participants were asked only to cut and suture precisely along two inner curves $(C)$. Each curve on the left and right sides of the central circle $(C)$ was three quarters of a 4-mm radius circle. Finally, the nine small points (see the red arrow in Fig. 2) in the model were used to keep participants' attention focused on the centre and also, we used these points for the eye tracking calibration procedure.

We provided a 7-0 suturing material, $20 \mathrm{~cm}$ thread and suitable micro-instruments (i.e. scissors, needle holder and forceps) for all participants (Fig. 3).

\section{Participants}

Nine (seven male and two female) neurosurgeons with varying years of training and experience participated in this experiment (Table 1). All participants, except one, 


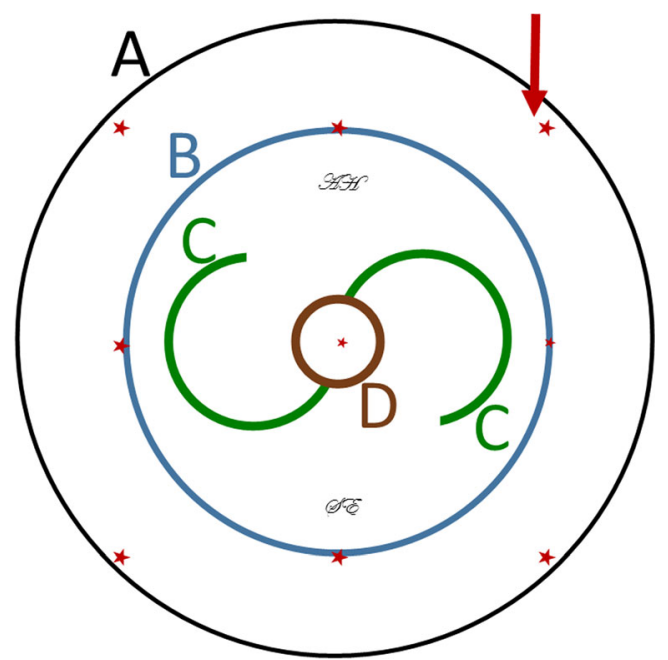

Fig. 2 Schematic view of cutting and suturing tasks. The two outer circles $(A$ and $B)$ represent the field of view under the microscope when using two different magnifications. Participants were asked to cut and suture precisely along two inner green lines $(C)$ on the left and right sides of central circle $(D)$. Radius: $A=15 \mathrm{~mm}, B=10 \mathrm{~mm}, C=4 \mathrm{~mm}$ and $D=2 \mathrm{~mm}$

were right handed. Four participants using eyeglasses were asked to adjust the diopter scale of the microscope eyepieces and work without glasses (the light reflected from glasses affects the performance of the eye tracker).

Regarding of their years of training experience and working hours, we assigned the surgeons into the two groups based on their average years of experience and working hours ( 5 years, $5 \mathrm{~h} /$ week). As a result, three participants belonging to the expert group and six participants to the novice group (Table 1).

\section{Eye tracking}

To capture the neurosurgeons' eye movements, we extended Eivazi et al.'s [11] system to include two glints (cornea reflection to the light) for head movement compensation. Three Ps3Eye cameras with a sampling rate of $60 \mathrm{~Hz}$ were used to record left and right eye movements (eye cameras) and the microscope field of view (scene camera). All three videos were simultaneously captured using iPi recorded software (version 2.2.3.30; iPi Soft, www.ipisoft.com).

It is beyond both the scope and purpose of this paper to offer a coverage of the eye tracking principle used in this study (see Duchowski [7] and Hansen [17] for more detailed information on the video-oculography (VOG) eye tracking principle). In summary, Fig. 4 shows the three key steps to determine the gaze points using the VOG method.

\section{Experiment procedure}

First, we explained the experiment and the tasks for all participants. Subsequently, participants provided their individual related information (Fig. 3). The warm-up task was designed to prepare participants for recording tasks. The actual recording started with a cutting task, suturing the left side and suturing the right side of the model (with a short break in between). Participants were instructed to keep their eyes fixed on the microscope ocular during the experiment, even when handling the instruments; the nine point calibrations were repeated at the start and the end of each task.
Fig. 3 The actual experiment design with micro-instrument. To evaluate our task design, we asked participants to rate our model from 0 to 5 , considering 5 a maximum. The average task design satisfaction score was 4.4 (SD, 0.5)

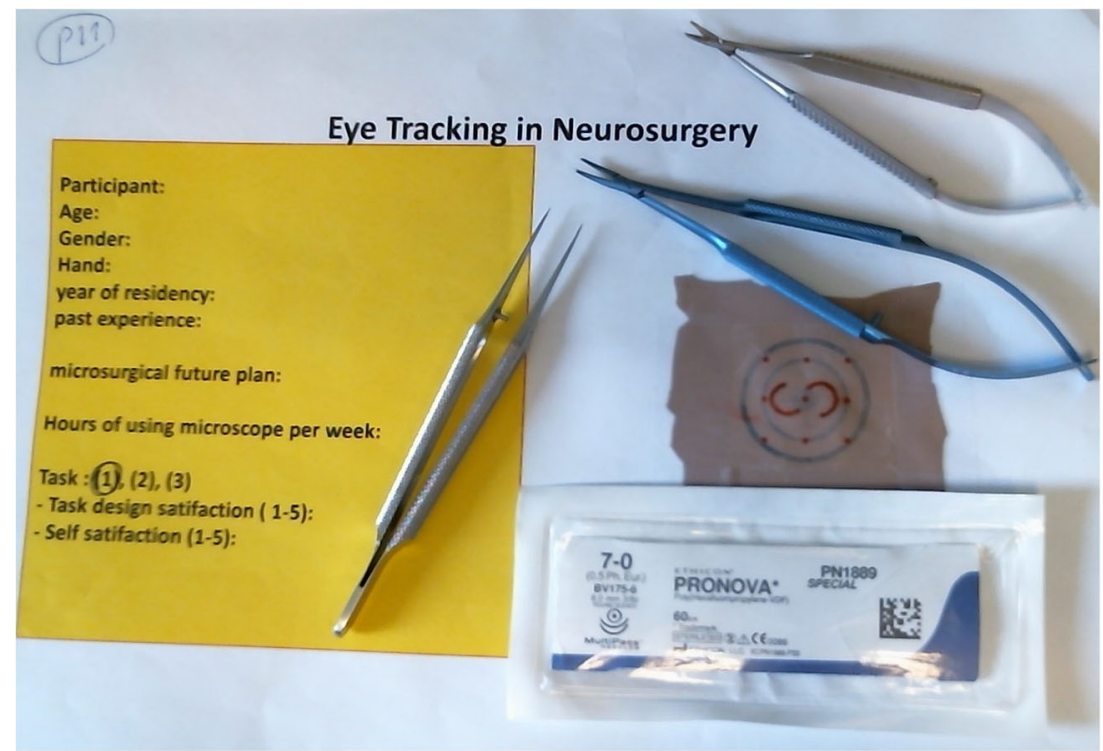


Table 1 Participants' expertise details [average (standard deviation)]

Age Years of Weekly hours Total hours
experience (of using microscope) in OR

\begin{tabular}{lllrc}
\hline Participants & $33(4)$ & $5(3)$ & $5(3)$ & $1,251(1,602)$ \\
Novices & $31(4)$ & $3(1)$ & $3.5(2)$ & $513(434)$ \\
Experts & $38(2)$ & $8(3)$ & $7(3)$ & $2,728(2,214)$
\end{tabular}

We estimated the total hours using the microscope in OR by multiplying 44 weeks of the year with weekly work hours

\section{Data analysis}

The data were analysed using Microsoft Excel and Matlab tools. The eye movement analysis is based on two principal measures of visual attention: fixation and saccade [21]. Fixation refers to an event when eyes are focused on an object in a scene. A saccade, on the other hand, occurs when the eyes quickly change focus from one place in space to other.

We employed a velocity-based algorithm for fixation and saccade identification [34]. The velocity threshold was set to 20 degrees/s, the minimum fixation duration was set to $100 \mathrm{~ms}$, and maximum distance between two gaze points was set to 20 pixels.

\section{Results}

We used mixed-model ANOVA analysis to show how eye movement patterns differ among novice and expert surgeons. We compared the mean differences between groups that have been split based on two factors, where expertise level is a between-subject factor and the experiment session is a within-subjects factor. For instance, in the suturing task each stitch considered as a within-subjects factor.

\section{Cutting task}

On average, participants spent 1:43 min on the cutting task, in which $50 \mathrm{~s}$ was spent on the left side and $46 \mathrm{~s}$ on the right of our model. The ANOVA analysis revealed significant differences in the expertise level for the average fixation duration $\left(\mathrm{F}[1,15]=6.29, p=0.03, \eta^{2}=0.26\right)$. The differences in the fixation rate were not significant, but considerably strong $\left(\mathrm{F}[1,15]=3.88, p=0.08, \eta^{2}=0.22\right)$.

We could not find any statistical differences in the saccades' features regarding the expertise groups for this task. Moreover, in all cases, the experiment sessions had no effects on the fixations and saccades, and there was no interaction effect between the expertise and experiment sessions.

Table 2 shows the comparison between eye movement features and the level of training. In the cutting task, neurosurgeons were able to focus continuously on the task without distractions (swapping instruments). Experts had longer fixation durations with a correlation coefficient of 0.3 . We also see the same effect of expertise on the fixation rate. This means experts had fewer fixations with a longer fixation duration than novices.

What is more significant here is the fixation duration differences in the left and right sides of the model. It is known that the left side is more difficult because all surgeons but one were right handed. Moreover, it took longer for participants to complete the left-side task than the right side (however, the $t$ test results led us to not reject the null hypothesis). For the lefthanded participant, in contrast, a longer fixation duration was related to the right side of the model ( $35 \mathrm{~ms}$ difference).

\section{Suturing task}

On average, surgeons spent 20:43 min on the suturing task. They had a short break between each side of the model.

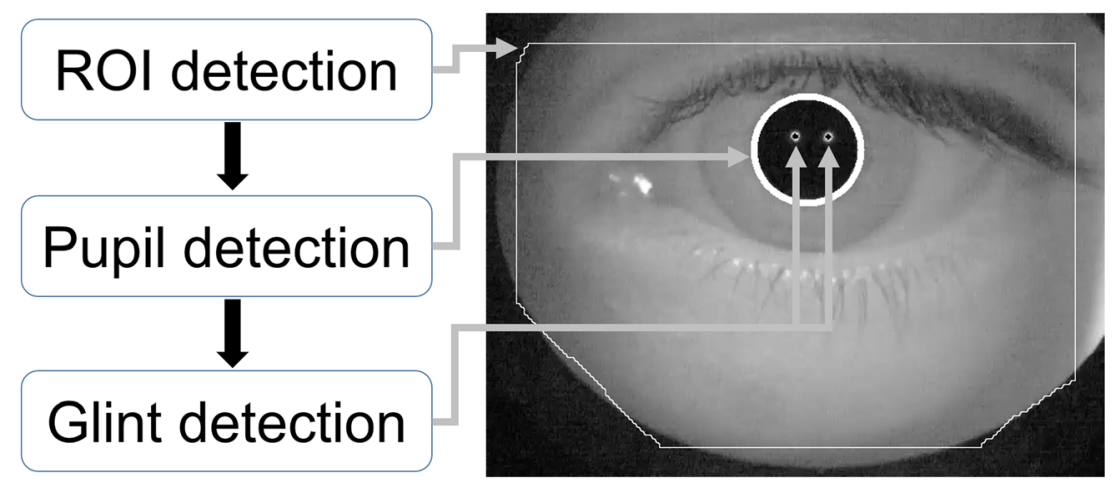

Fig. 4 Three key steps to determine a gaze point based on the video-oculography (VOG) method. First, for the detection of the region of interest (ROI), our algorithm downscales the image and then calculates the mean grey value. After an ROI is found, the algorithm $[25,26]$ searches for the image area that represents the pupil and glint points. During a calibration procedure, we calculate the vector between the pupil centre and the centre of both glints. Then, a least-squares polynomial fit is calculated where the vectors are input variables and the calibration point positions are the polynomial result. After calibration, the new pupil glint vectors and the polynomials are used for the gaze position estimation 
Table 2 Comparison between eye movement features and the level of training when surgeons participated in the cutting task

\begin{tabular}{llllll}
\hline Eye movement metrics) & \multicolumn{2}{l}{ Right side } & & \multicolumn{2}{l}{ Left side } \\
\cline { 2 - 3 } \cline { 5 - 6 } & Experts & Novice & & Experts & Novice \\
\hline Fixation duration (ms) & $500(188)$ & $382(197)$ & & $848(377)$ & $402(185)$ \\
Fixation rate & $0.0022(0.0008)$ & $0.0030(0.001)$ & & $0.0013(0.0006)$ & $0.0030(0.001)$ \\
Saccade duration (ms) & $32(12)$ & $36(14)$ & & $26(6)$ & $39(15)$ \\
Saccade rate & $0.033(0.01)$ & $0.031(0.01)$ & & $0.039(0.009)$ & $0.029(0.008)$ \\
Saccade amplitude (degree) & $0.7(0.4)$ & $0.6(0.2)$ & & $0.5(0.2)$ & $0.6(0.1)$ \\
\hline
\end{tabular}

The measures are presented as average (standard deviation)
Participants spent 18:39 min on the left side of the model and 10:17 on the right side (however, the $t$-test results led us to not reject the null hypothesis). This might indicate that suturing the left side was more challenging for the right handedparticipants.

The average fixation duration for the suturing task shows a similar trend as the cutting task (longer fixation duration for experts); however, none of the effects was significant, and the differences between experts and novices were weaker compared to the cutting task.

There were significant differences for expertise level's effect on the saccade duration $(\mathrm{F}[1,52]=9.43, p=0.004$, $\left.\eta^{2}=0.16\right)$, saccade rate $(\mathrm{F}[1,52]=10.22, p=0.003$, $\left.\eta^{2}=0.17\right)$, and saccade amplitude $(\mathrm{F}[1,52]=4.78$, $p=0.03, \eta^{2}=0.09$ ) when suturing the right side of the model.

In the suturing left side, the expertise effect was strong for the saccade amplitude $\left(\mathrm{F}[1,52]=5.33, p=0.03, \eta^{2}=0.10\right)$, but not for saccade duration and saccade rate. In all cases, the experiment session (location of sutures) had no effects on the fixation and saccade features, and there was no interaction effect between the expertise and experiment sessions. Table 3 shows the comparison between eye movement features and the level of training for the suturing task.

Compared to the cutting task, suturing is more complex and includes more distractions (e.g. swapping instruments), and thus experts were not able to apply long fixations in this task. However, experts changed their saccade strategy by having shorter saccades with longer amplitudes compared to novices for the right-side task. Finally, in the left-side task, experts' saccade amplitude reversed.

\section{Discussion}

In this study, we used eye tracking technology to quantify neurosurgeons focus continuity with hand movements when performing different training tasks using a neurosurgical microscope. Visual attention and eye-hand coordination skills are essential for developing and mastering surgical techniques $[14,27,36,40]$. In micro-neurosurgery, moreover, the learning curve for eye-hand coordination is long and may depend on subjective psychomotor abilities. Surgeons have to learn to scan across the operating field while concentrating on tiny objects in the operating field. As such, they have to maintain attention to follow the procedure and avoid obstacles while safely moving the instrument through delicate structures.

In this study, we found that in spite of certain neurosurgeon-to-neurosurgeon variability, there were statistically significant differences in eye movement patterns between participants, as well as different tasks. For instance, in the cutting task, the attention (centre of gaze) of all neurosurgeons was inevitably directed to the area in which the tip of the scissors meets the cutting-angle gape. Therefore, the important spot in the cutting field acts as a low-level visual cue (Fig. 5).

Moreover, the gaze analysis revealed a long fixation duration and high level of focus for the experts in this task. In the cutting task, well-trained neurosurgeons develop their fixation duration on the operating field up to twice as much as novices.

We then studied the eye reacting pattern in the suturing task; compared to the cutting task, there was a larger variety across subjects and more complex manipulation with the centre of gaze, although there was a similar pattern of response.

We showed how simple distractions, such as swapping instruments, needle falls or loose thread [10] could disturb the delicate harmony of composition, biasing the eyes away from the intended task. Surgeons have no choice but to accept the shorter fixation duration as a form of optimal strategy and then change their visual search strategy (saccades) for further information processing.

To overcome the problem with distractions and reduced concentration level in our experiment, experts took a different strategy. For example, they began to apply shorter and more frequent saccades when suturing the right side of our model. This means a skilled neurosurgeon must maintain a precise eye-hand span and hold a large amount of information more frequently from the certain surgical procedure or field.

Tying these findings back to prior visual attention studies in the field of science $[13,21,32]$ as well as prior work on eye tracking in surgery $[19,36]$, we see that some of these strategies are undoubtedly unique to micro-neurosurgery, both in terms of fixations as well as saccades. Maintaining steady 
Table 3 Comparison between eye movement features and the level of training when surgeons participated in the suturing task

\begin{tabular}{llllll}
\hline Eye movement metrics & \multicolumn{2}{l}{ Right side } & & Left side & \\
\cline { 2 - 3 } \cline { 5 - 6 } & Experts & Novice & & Experts & Novice \\
\hline Fixation duration (ms) & $318(83)$ & $305(132)$ & & $332(117)$ & $296(110)$ \\
Fixation rate & $0.0033(0.0008)$ & $0.0037(0.001)$ & & $0.0034(0.001)$ & $0.0038(0.001)$ \\
Saccade duration (ms) & $31(4)$ & $36(5)$ & & $37(6)$ & $34(6)$ \\
Saccade rate & $0.033(0.005)$ & $0.028(0.004)$ & & $0.029(0.005)$ & $0.031(0.005)$ \\
Saccade amplitude (degree) & $0.6(0.1)$ & $0.7(0.1)$ & & $0.8(0.1)$ & $0.7(0.1)$ \\
\hline
\end{tabular}

The measures are presented as average (standard deviation) visual attention on the target (fixation), as well as being able to quickly make eye jumps from one target to another (saccades) are two important elements for the success of such a surgery.

The longer fixation duration for experts in microneurosurgery can be explained with the informationreduction hypothesis $[15,16]$. This theory proposes that experts learn, with practice, to distinguish between task-relevant and redundant information and to limit their information processing to the relevant information. We believe that professional neurosurgeons learned the selectivity of visual information processing so that they are able to chunk several notes together and process them as a single unit of information.

Moreover, changes in the saccade measures may show the increase of interactions between the perception and action systems. During eye-hand coordination, the visual information system optimises saccades for target localisation and accuracy feedback of sensimotor system. In micro-neurosurgery, the small and narrow fields of view elevate the difficulty of

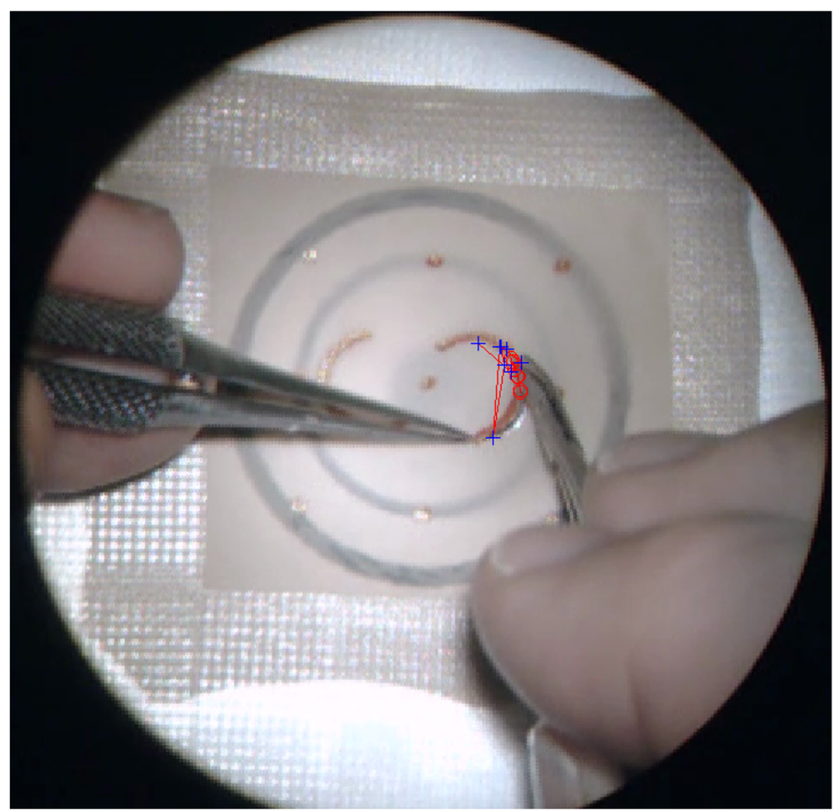

Fig. 5 An example of 2-s scan-path of the fixations (red circles) and saccades (blue cross). The attention (fixation) of the neurosurgeon is attracted to the area in the field where the tip of the scissors meets the angle gape of cutting information processing. We believed the special direction between eyes and hands and the small size of target affect eye movements and thus experts apply various saccade patterns to overcome these challenges. For example, changes in saccade amplitude could occur when the target was covered by hands or instruments.

\section{Implications for neurosurgical training systems}

One area of significant opportunity is to integrate our technology in new neurosurgical training simulators. For example, incorporation of gaze tracking into the NeuroTouch simulator platform (National Research Council Canada, http:/www. neurotouch.ca/eng/partners.html).

In this paper, we study only eye behaviour as a parameter for hand movement under the microscope. Our trajectory, here, was from eye to hand. In a following-up study, we plan to use eye-hand coordination metrics to study the relationship between non-achievement hand movements with disturbance of the eyes and focus. In future work, we will apply eye movements tracking alongside with hand-motion measuring and force-sensing capability methods [18].

The established metrics that measure trainees' progress or milestone accomplishments can then serve as a guide for the supervisors to change training priorities dynamically and pinpoint specific tasks that trainees need to accomplish at each transition stage. Therefore, we suggest cooperation with microscope companies to develop an eye tracker to all neurosurgical microscopes that gives the feedback after each procedure to the neurosurgeon to study eye-hand behaviour.

A second consideration here is to apply these finding to the eye-hand training of neurosurgeons (see examples in golf training [38] or laparoscopic training [39]). For example, by designing a goal-directed aiming task for residents and then track eye, hand and instrument movements to evaluate the trainee's performance to determining whether a specified eye movement pattern was met. Such gaze training tasks would be beneficial for two main reasons. First, residents may learn where and what to focus on, thus replaying the gaze behaviour of experts, may help novices detect the importance of these areas. Second, trainees may learn about which pattern to focus 
on, thus modelling the patterns of visual search may be used as perceptual cues on how to navigate in such an environment.

\section{Limitations}

One limitation is that our data samples are small and limited to Helsinki University Hospital [20, 28]. Moreover, the environment characteristics should be taken into account in generalising these results.

The suturing and cutting test designed in this study is a narrow task compared with neurosurgery practices as a whole. A further potential limitation is the fact that we added an eye tracker to the surgical microscope, which limits the field of view. Participant performance may have been affected by the presence of the eye tracker or specific setting we designed in this study; namely, the type of microscope, instruments or not letting participant use their glasses.

The number of participants involved in this study need to be increased and balance, so that we will be able to divide participants in three groups: junior trainees, senior trainees and senior neurosurgeons.

Therefore, the current analysis represents the first step towards improving our understanding of expertise differences in the field of micro-neurosurgery. Our future aim is to expand our data samples by conducting our experiment in various neurosurgical lab and surgical practices with more advanced scan-path analysis $[8,35]$.

Acknowledgements We would like to thank all surgeons who participated in this study, and Helsinki University Hospital for enabling us to conduct this experiment.

\section{Compliance with ethical standards}

Funding No funding was received for this research.

\section{Conflict of interest None.}

Ethical approval For this type of study formal consent is not required.

Informed consent Informed consent was obtained from all individual participants included in the study.

\section{References}

1. Afkari H, Eivazi S, Bednarik R, Mäkelä S (2014) The potentials for hands-free interaction in micro-neurosurgery. In: Proceedings of NordiCHI, Helsinki, 26-30 October 2014, pp 401-410. doi:10. 1145/2639189.2639213

2. Afkari H, Bednarik R, Mäkelä S, Eivazi S (2016) Mechanisms for maintaining situation awareness in the micro-neurosurgical operating room. Int J Hum Comput Stud 95:1-14

3. Atkins MS, Tien G, Khan RS, Meneghetti A, Zheng B (2013) What do surgeons see capturing and synchronizing eye gaze for surgery applications. Surg Innov 20(3):241-248
4. Bourne SK, Walcott BP, Sheth SA, Coumans JVC (2013) Neurological surgery: the influence of physical and mental demands on humans performing complex operations. J Clin Neurosci 20(3):342-348

5. Chan WY, Matteucci P, Southern SJ (2007) Validation of microsurgical models in microsurgery training and competence: a review. Microsurgery 27(5):494-499

6. Chan W, Niranjan N, Ramakrishnan V (2010) Structured assessment of microsurgery skills in the clinical setting. J Plast Reconstr Aesthet Surg 63(8):1329-1334

7. Duchowski A (2007) Eye tracking methodology, theory and practice, 2nd edn. Springer, London

8. Eivazi S, Bednarik R (2011) Predicting problem-solving behavior and performance levels from visual attention data. In: Proceedings of the 2nd Workshop on Eye Gaze in Intelligent Human Machine Interaction at IUI,, Palo Alto, 13 February 2011, pp 9-16

9. Eivazi S, Bednarik R, Tukiainen M, von und zu Fraunberg M, Leinonen V, Jääskeläinen JE (2012) Gaze behaviour of expert and novice microneurosurgeons differs during observations of tumor removal recordings. In: Proceedings of the ACM Symposium on Eye Tracking Research and Applications, ETRA, Santa Barbara, 28-30 March 2012, pp 377-380. doi:10.1145/2168556.2168641

10. Eivazi S, Afkari H, Bednarik R, Leinonen V, Tukiainen M, Jääskeläinen JE (2015) Analysis of disruptive events and precarious situations caused by interaction with neurosurgical microscope. Acta Neurochir (Wein) 157(7):1147-1154

11. Eivazi S, Bednarik R, Leinonen V, von und zu Fraunberg M, Jääskeläinen JE (2016) Embedding an eye tracker into a surgical microscope: requirements, design, and implementation. IEEE Sensors J 16(7):20-70

12. Fanua SP, Kim J, Shaw Wilgis E (2001) Alternative model for teaching microsurgery. Microsurgery 21(8):379-382

13. Gegenfurtner A, Lehtinen E, Säljö R (2011) Expertise differences in the comprehension of visualizations: a meta-analysis of eyetracking research in professional domains. Educ Psychol Rev 23(4):523-552

14. Grober ED, Hamstra SJ, Wanzel KR, Reznick RK, Matsumoto ED, Sidhu RS, Jarvi KA (2003) Validation of novel and objective measures of microsurgical skill: hand-motion analysis and stereoscopic visual acuity. Microsurgery 23(4):317-322

15. Haider H, Frensch PA (1996) The role of information reduction in skill acquisition. Cogn Psychol 30(3):304-337

16. Haider H, Frensch PA (1999) Eye movement during skill acquisition: more evidence for the information-reduction hypothesis. J Exp Psychol Learn Mem Cogn 25(1):172-190

17. Hansen DW, Ji Q (2010) In the eye of the beholder: a survey of models for eyes and gaze. IEEE Trans Pattern Anal Mach Intell 32(3):478-500

18. Harada K, Morita A, Minakawa Y, Baek YM, Sora S, Sugita N, Kimura T, Tanikawa R, Ishikawa T, Mitsuishi M (2015) Assessing microneurosurgical skill with medico-engineering technology. World Neurosurg 84(4):964-971

19. Hermens F, Flin R, Ahmed I (2013) Eye movements in surgery: a literature review. J Eye Mov Res 6(4):1-11

20. Hernesniemi J, Niemelä M, Karatas A, Kivipelto L, Ishii K, Rinne J, Ronkainen A, Koivisto T, Kivisaari R, Shen H, Lehecka M, Frösen J, Piippo A, Jääskeläinen JE (2005) Some collected principles of microneurosurgery: simple and fast, while preserving normal anatomy: a review. Surg Neurol 64:195-200

21. Holmqvist K, Nyström M, Andersson R, Dewhurst R, Jarodzka H, Van de Weijer J (2011) Eye tracking: a comprehensive guide to methods and measures. Oxford University Press, Oxford

22. Ibbotson JA, MacKenzie CL, Cao CGL, Lomax AJ (1999) Gaze patterns in laparoscopic surgery. Stud Health Technol Inform 62: $154-160$ 
23. Kübler TC, Eivazi S, Kacneci E (2015) Automated visual scanpath analysis reveals the expertise level of micro-neurosurgeons. In: Workshop on Interventional Microscopy at International Conference on Medical Image Computing and Computer-Assisted Interventions (MICCAI), 5-9 October 2015, Munich. https://goo.gl/ 4780eE. Accessed 7 March 2017

24. Kundel HL, La Follette PS Jr (1972) Visual search patterns and experience with radiological images 1. Radiology 103(3):523-528

25. Kundel HL, Nodine CF, Carmody D (1978) Visual scanning, pattern recognition and decision-making in pulmonary nodule detection. Investig Radiol 13(3):175-181

26. Lahiri A, Lim AY, Qifen Z, Lim B (2005) Microsurgical skills training: a new concept for simulation of vessel-wall suturing. Microsurgery 25(1):21-24

27. Law B, Atkins MS, Kirkpatrick AE, Lomax AJ (2004) Eye gaze patterns differentiate novice and experts in a virtual laparoscopic surgery training environment. In: Proceedings of ETRA, ACM 4148. doi:10.1145/968363.968370

28. Lehecka M, Laakso A, Hernesniemi J, Çelik Ö (2011) Helsinki Microneurosurgery basics and tricks. Druckerei Hohl, Balgheim

29. Nodine CF, Kundel HL (1987) Using eye movements to study visual search and to improve tumor detection. Radiographics 7(6): $1241-1250$

30. Nowitzke AM (2005) Assessment of the learning curve for lumbar microendoscopic discectomy. Neurosurgery 56(4):755-762

31. Ramachandran S, Ghanem AM, Myers SR (2013) Assessment of microsurgery competency - where are we now? Microsurgery 33(5):406-415

32. Rayner K (1998) Eye movements in reading and information processing: 20 years of research. Psychol Bull 124(3):372-422

33. Richstone L, Schwartz MJ, Seideman C, Cadeddu J, Marshall S, Kavoussi LR (2010) Eye metrics as an objective assessment of surgical skill. Ann Surg 252(1):177-182

34. Salvucci DD, Goldberg JH (2000) Identifying fixations and saccades in eye-tracking protocols. In: Proceedings of the ACM Symposium on Eye Tracking Research and Applications, ETRA, Santa Barbara, 28-30 March 2012, pp 71-78. doi:10.1145/355017. 355028

35. Tafaj E, Kasneci G, Rosenstiel W, Bogdan M (2012) Bayesian online clustering of eye movement data. In: Proceedings of the ACM Symposium on Eye Tracking Research and Applications,
ETRA, Santa Barbara, 28-30 March 2012, pp 285-288. doi:10. 1145/2168556.2168617

36. Tien T, Pucher PH, Sodergren MH, Sriskandarajah K, Yang GZ, Darzi A (2014) Eye tracking for skills assessment and training: a systematic review. J Surg Res 191(1):169-178

37. Tomizawa Y, Aoki H, Suzuki S, Matayoshi T, Yozu R (2012) Eyetracking analysis of skilled performance in clinical extracorporeal circulation. J Artif Organs 15(2):146-157

38. Vine SJ, Moore L, Wilson MR (2011) Quiet eye training facilitates competitive putting performance in elite golfers. Front Psychol 2:816

39. Vine SJ, Masters RS, McGrath JS, Bright E, Wilson MR (2012) Cheating experience: guiding novices to adopt the gaze strategies of experts expedites the learning of technical laparoscopic skills. Surgery 152(1):32-40

40. Wilson M, McGrath J, Vine S, Brewer J, Defriend D, Masters R (2010) Psychomotor control in a virtual laparoscopic surgery training environment: gaze control parameters differentiate novices from experts. Surg Endosc 24(10):2458-2464

\section{Comments}

Microsurgical skill acquisition at an earlier stage of neurosurgery training represents an essential goal to reach an objective competence-based assessment. The analysis of the gaze patterns using eye tracking technology to quantify neurosurgeons' focus continuity movements performing different training tasks using a neurosurgical microscope is a valuable idea. The collected data can be used to design special tasks to develop longer fixation duration or to increase the interaction between perception and action systems. Moreover, the subjective psychomotor abilities will enhance more with learning to concentrate on the objective field, reducing unnecessary distractions and optimising the microsurgical strategy. The opportune introduction of these evidences in the training processes or to integrate the technology in new neurosurgical trainingsimulators can reduce the learning curve of difficult surgical techniques, accelerate the rate for trainees to achieve surgical competency and improve patient safety.

Alex Alfieri, Lehel Török,

Brandenburg, Germany 